\title{
Hiperactividad simpática paroxística: prevalencia, tratamiento y hallazgos neurorradiológicos en pacientes pediátricos
}

\author{
Antonio Bravo-Oro ${ }^{1}$, Héctor Gerardo Hernández-Rodríguez², Jorge Guillermo Reyes-Vaca ${ }^{3}$, \\ Miguel Villegas-Aguilera ${ }^{1}$, Claudia Lizbeth Gómez-Elías ${ }^{1}$, Rosalina Rivera-Vega ${ }^{4}$, \\ Luís Fernando Mendoza-Moreno ${ }^{4}$ y Jorge Luís García-Ramírez ${ }^{1}$ \\ ${ }^{1}$ Departamento de Neurología Pediátrica, Hospital Central Dr. Ignacio Morones Prieto; ${ }^{2}$ Departamento de Epidemiología, Universidad Autónoma \\ de San Luis Potosi; ${ }^{3}$ Departamento de Radiología; ${ }^{4}$ Departamento de Cuidados Intensivos Pediátricos. Hospital Central Dr. Ignacio Morones \\ Prieto, San Luis Potosí, México
}

\begin{abstract}
Resumen
Introducción: La hiperactividad simpática paroxística (HSP) es un trastorno poco estudiado y comprendido en pediatría, y se asocia principalmente a traumatismo craneoencefálico (TCE) e hipoxia. El cuadro clínico se caracteriza por episodios autolimitados y recurrentes de hipertensión, taquicardia, taquipnea, hipertermia, sialorrea, midriasis, hiperhidrosis, disminución del nivel de conciencia y aumento del tono muscular con postura en extensión. En edad pediátrica se estima una prevalencia del 12-29\%. Objetivo: Determinar la prevalencia del padecimiento en nuestra institución, la etiología, el tratamiento utilizado para su manejo y los hallazgos neurorradiológicos. Método: Se realizó un estudio retrospectivo con los pacientes internados en el periodo comprendido entre enero de 2002 y enero de 2017 en cuidados intensivos pediátricos. Se identificaron siete casos que cumplieron los criterios diagnósticos de HSP. Resultados: La media de edad fue de 1 a 14 años y la etiología más frecuente estuvo asociada a paro cardiorrespiratorio. Iniciaron con el trastorno en un promedio de 21.74 días $(D E \pm 18.37)$, el tratamiento se inició en un promedio de 2.00 días $(D E \pm 2.07)$ y el tiempo de internamiento estuvo en un rango de 20-180 días -días de estancia en terapia con un rango de 10-40 días. Los fármacos utilizados para el control del padecimiento fueron propranolol (PPN), baclofeno, buprenorfina (BPN) y gabapentina (GBP), con los que se logró el control en un promedio de 5.14 días (desviación estándard $(D E) \pm 2.17$ ). Los hallazgos más frecuentes en resonancia magnética fueron lesiones hipóxico-isquémicas en sustancia blanca, núcleos de la base y tálamos. Conclusiones: La HSP es un padecimiento grave que se asocia a una alta morbimortalidad. La prevalencia en nuestra serie fue del $1.12 \%$, muy baja si se compara con lo reportado por otros autores. Se debe realizar un consenso para establecer los criterios diagnósticos y el tratamiento en la población pediátrica.
\end{abstract}

Palabras clave: Hiperactividad simpática paroxística. Unidad de Cuidados Intensivos Pediátricos. Resonancia magnética. Diencéfalo.

\section{Correspondencia:}

Antonio Bravo-Oro

Departamento de Neurología Pediátrica

Hospital Central Dr. Ignacio Morones Prieto

Avda. Venustiano Carranza, 2395

Fecha de recepción: 08-01-2018

Fecha de aceptación: 13-09-2018

DOI: $10.24875 /$ RMN.M18000012
Disponible en internet: 03-01-2019 Rev Mex Neuroci. 2018;19(6):3-11 www.revmexneurociencia.com

E-mail: antoniobravooro@ hotmail.com 1665-5044/@ 2018. Academia Mexicana de Neurología A.C. Publicado por Permanyer México. Este es un artículo Open Access bajo la licencia CC BY-NC-ND (http://creativecommons.org/licenses/by-nc-nd/4.0/). 


\title{
Paroxysmal sympathetic hyperactivity: prevalence, treatment and neuroradiological findings in pediatric patients
}

\begin{abstract}
Objective: Paroxysmal sympathetic hyperactivity is a disorder poor studied and understood in pediatrics. It is mainly associated with cranioencephalic trauma and hypoxia. Is characterized by self-limited and recurrent episodes of hypertension, tachycardia, tachypnea, hyperthermia, sialorrhea, mydriasis, hyperhidrosis, decreased level of consciousness and increased muscle tone with extension posture. In pediatric age, a prevalence of $12-29 \%$ is estimated. Methods: A retrospective study was conducted with patients admitted in the period from January 2002 to January 2017 in pediatric intensive care. Seven cases met the diagnostic criteria of paroxysmal sympathetic hyperactivity. Results: The mean age was 1-14 years, the most frequent etiology was associated with cardiorespiratory arrest. They started with the disorder in an average of 21.74 days (standard deviation [SD] 18.37), the treatment started on average of 2.00 days (SD 2.07) and internment time in a range of 20-180 days, therapy stay with a range of 10-40 days. The drugs used to control the condition were propranolol, baclofen, buprenorphine and gabapentin, achieving control on average of 5.14 days (SD 2.17). The most frequent findings in magnetic resonance were hypoxic ischemic lesions in white matter, basal ganglia and thalami. Conclusions: Paroxysmal sympathetic hyperactivity is a serious condition associated with high morbidity and mortality. The prevalence in our series was $1.12 \%$, very low compared to that reported by other authors. A consensus must be established to establish diagnostic criteria and treatment in the pediatric population.
\end{abstract}

Key words: Paroxysmal sympathetic hyperactivity. Pediatric Intensive Care Unit. Magnetic resonance. Diencephalon.

\section{Introducción}

La disfunción autonómica en pacientes pediátricos secundaria a lesiones en el sistema nervioso central (SNC) es un trastorno poco estudiado, por lo que también es poco comprendido. El cuadro clínico se caracteriza por la aparición de episodios autolimitados y recurrentes con una duración variable de manifestaciones autonómicas de hipertensión, taquicardia, taquipnea, hipertermia, sialorrea, midriasis, hiperhidrosis y disminución del nivel de conciencia, junto con manifestaciones motoras de hipertonía con postura en extensión ${ }^{1,2}$. Se asocia principalmente a TCE, accidente vascular, hipoxia, hemorragia subaracnoidea, lesiones medulares, tumores supraselares o diencefálicos, encefalitis autoinmune, hidrocefalia y neuroinfección (NI) ${ }^{3-7}$.

Strich, en 1956, fue el primero en describir un caso con manifestaciones autonómicas, que denominó crisis del tallo cerebral ${ }^{8}$. Se han utilizado diferentes nombres, como crisis diencefálicas, inestabilidad autonómica paroxística con distonía, síndrome disfuncional del tallo cerebral y tormenta paroxística simpática, pero a partir del consenso de 2014 se decidió utilizar el término hiperactividad simpática paroxística (HSP) ${ }^{9}$. En este mismo consenso se establecieron los criterios diagnósticos y se concluyó que deben estar presentes las siguientes 11 manifestaciones clínicas: a) ocurren todas las manifestaciones clínicas al mismo tiempo, b) los episodios son de naturaleza paroxística, c) sobreactividad simpática con estímulos normales no dolorosos, d) persisten por $\geq 3$ días consecutivos, e) persisten por $\geq 2$ semanas por daño cerebral, f) persisten a pesar de tratar otros diagnósticos diferenciales, g) se administraron medicamentos para disminuir las manifestaciones simpáticas, h) $\geq 2$ eventos al día, i) ausencia de manifestaciones parasimpáticas durante los eventos, j) ausencia de otras causas de las manifestaciones y k) antecedente de daño cerebral ${ }^{9}$.

Se ha diseñado una herramienta para evaluar a los pacientes desde la admisión a la Unidad de Cuidados Intensivos hasta la rehabilitación denominada la medida de evaluación (PSH-AM), la cual está conformada por dos componentes: uno que evalúa el grado de severidad de las características clínicas (escala de manifestaciones clínicas [CFS]) y otro que establece la probabilidad del diagnóstico (herramienta de probabilidad diagnóstica [DLT]). La CFS incluye las 6 manifestaciones cardinales del padecimiento (taquicardia, taquipnea, hipertensión, hipertermia, sudoración y posturas durante los episodios) ${ }^{9}$ y la DLT los 11 ítems de diagnóstico señalados anteriormente. La suma de estos dos componentes estima la probabilidad diagnóstica de HSP en ese momento (Tabla 1).

La prevalencia de la HSP es muy variable: en adultos se reporta del 8 al $33 \%$ asociada a TCE y del 6 al 29\% secundaria a hipoxia. Respecto a los casos en edad pediátrica, no se cuenta con muchos reportes, pero se estima una prevalencia del $12 \%$ asociada a TCE y del 
Tabla 1. Medida de evaluación (PSH-AM) ${ }^{9}$

\begin{tabular}{|l|c|c|c|c|c|}
\hline \multicolumn{1}{|l|}{ CFS } \\
\hline & $\mathbf{0}$ & $\mathbf{1}$ & $\mathbf{2}$ & $\mathbf{3}$ & Calificación \\
\hline FC & $<100$ & $100-119$ & $120-139$ & $\geq 140$ \\
\hline FR & $<18$ & $18-23$ & $24-29$ & $\geq 180$ \\
\hline TA sistólica & $<140$ & $140-159$ & $160-179$ & $\geq 39.0$ \\
\hline Temperatura & $<37$ & $37-37.9$ & $38-38.9$ & Severa \\
\hline Sudoración & No & Leve & Moderada & Severa \\
\hline Postura & No & Leve & Moderada & CSF Total \\
\hline
\end{tabular}

FC: Frecuencia cardíaca; FR: Frecuencia respiratoria; TA: Tensión arterial; Grado de severidad: Nulo = 0; Leve = 1-6; Moderado = 7-12; Severo $\geq 13$.

DLT

Las características clínicas ocurren al mismo tiempo

Los episodios son de naturaleza paroxística

Sobreactividad simpática con estímulos normales no dolorosos

Las características persisten por $\geq 3$ días consecutivos

Las características persisten por $\geq 2$ semanas por insulto cerebral

Las manifestaciones persisten a pesar de tratar otros diagnósticos diferenciales

Se administraron medicamentos para disminuir las manifestaciones simpáticas

$\geq 2$ eventos al día

Ausencia de manifestaciones parasimpáticas durante los eventos

Ausencia de otras causas de las manifestaciones

Antecedente de daño cerebral

DLT total

1 punto por cada característica presente; Suma CFS + DLT; No probable $<8$; Posible = 8-16; Probable $>16$.

$29 \%$ posterior a lesiones hipóxicas, muy similar a lo descrito en adultos $3,10-13$.

Los mecanismos y las bases neuroanatómicas de la HSP aún no se comprenden bien del todo; la teoría más aceptada se basa en un desequilibrio del sistema nervioso autónomo provocado por una interrupción de la regulación cortical y pérdida del control del equilibrio autonómico por disfunción del hipotálamo y tálamo, que provoca una respuesta exagerada a los estímulos sensoriales inocuos y/o nociceptivos $^{14,15}$.

Más adelante describimos siete casos pediátricos con HSP analizando la etiología, los hallazgos neurorradiológicos y el tratamiento utilizado en la Unidad de Cuidados Intensivos Pediátricos (UCIP) del Hospital Central Dr. Ignacio Morones Prieto.

\section{Pacientes y métodos}

Se realizó un estudio retrospectivo con los pacientes internados en el periodo comprendido entre enero de 2002 y enero de 2017 en la UCIP del Hospital Central Dr. Ignacio Morones Prieto, con edades de 0 a 15 años. Se encontraron 2,933 internamientos, realizándose una búsqueda con los diagnósticos de ingreso de: TCE, NI, tumor en el SNC, accidente vascular cerebral (AVC) y 
Tabla 2. Características demográficas, etiología, días de internamiento, tratamiento y estado neurológico

\begin{tabular}{|c|c|c|c|c|c|c|c|}
\hline & Caso 1 & Caso 2 & Caso 3 & Caso 4 & Caso 5 & Caso 6 & Caso 7 \\
\hline Edad (años) & 14 años & 1 aก̃o & 5 años & 13 años & 11 años & 3 años & 14 años \\
\hline Sexo & M & M & $\mathrm{F}$ & M & $\mathrm{F}$ & M & M \\
\hline Mecanismo & TCE & $\mathrm{EHI}$ & $\mathrm{NI}$ & $\mathrm{EHI}$ & $\mathrm{EHI}$ & TCE & Tumor \\
\hline CFS & 3 & 3 & 3 & 3 & 3 & 3 & 3 \\
\hline Días de inicio de la HSP & 25 & 14 & 6 & 60 & 5 & 8 & 7 \\
\hline $\begin{array}{l}\text { Días de inicio del tratamiento } \\
\text { para la HSP }\end{array}$ & 1 & 2 & 1 & 1 & 1 & 7 & 1 \\
\hline Tiempo de internamiento & 65 & 40 & 65 & 180 & 26 & 32 & 20 \\
\hline Tiempo en la UCIP & 40 & 15 & 12 & 33 & 23 & 25 & 10 \\
\hline Tratamiento & PPN & Baclofeno & $\begin{array}{c}\text { Baclofeno } \\
\text { PPN } \\
\text { DEX }\end{array}$ & Baclofeno & $\begin{array}{c}\text { BPN } \\
\text { PPN } \\
\text { Baclofeno }\end{array}$ & $\begin{array}{c}\text { BPN } \\
\text { PPN } \\
\text { Baclofeno }\end{array}$ & $\begin{array}{l}\text { BPN } \\
\text { PPN } \\
\text { GBP }\end{array}$ \\
\hline Días sin HSP & 5 & 4 & 6 & 4 & 4 & 10 & 3 \\
\hline Escala de Rankin & 6 & 5 & 1 & 5 & 1 & 1 & 6 \\
\hline
\end{tabular}

PPN: Propranolol; DEX: Dexametasona; GBP: Gabapentin; BPN: Buprenorfina.

encefalopatía hipóxico-isquémica $(\mathrm{EHI})$ secundaria a paro cardiorrespiratorio. Se identificaron 627 casos.

Para el diagnóstico de HSP en este estudio se utilizaron los criterios establecidos en el consenso de $2014^{9}$, pero al tratarse de pacientes pediátricos se consideraron también signos vitales anormales y otras manifestaciones clínicas, según lo publicado por Kirk, et al. ${ }^{3}$. Éstas son: a) taquicardia por encima de $2 \mathrm{DE}$ para la edad, b) taquipnea cuando presentaba el doble de frecuencia para su edad, c) hipertensión con presión diastólica mayor del percentil 95 para la edad, d) fiebre $>38^{\circ} \mathrm{C}$, e) distonía (rigidez o postura de descerebración) diagnosticada por los médicos tratantes y f) diaforesis sin tener relación con otro tipo de padecimiento.

\section{Análisis estadístico}

Se utilizó el programa Excel 2010 como herramienta estadística. Los intervalos de confianza para la prevalencia encontrada se basaron en la distribución normal de probabilidad $p \pm 1.96$ error estándar para la prevalencia. Para la significancia estadística entre niños y niñas se utilizó la probabilidad exacta de Fischer, basada en la distribución hipergeométrica. Asimismo, para la significancia estadística en la comparación con otras prevalencias se empleó la distribución binomial de probabilidad.

\section{Resultados}

De los 627 casos revisados, 7 pacientes cumplieron los criterios de HSP, con una prevalencia del $1.12 \%$ (IC 95\%: 0.29-1.94). En el momento del diagnóstico la media de edad fue de 4 años y 10 meses (rango de 1 a 14 años); los pacientes eran 5 niños y 2 niñas, con una prevalencia no significativa del 1.17\% (IC 95\%: $0.15-2.2$ ) y del $1.01 \%$ (IC 95\%: 0.00-2.4), respectivamente $(p=0.61)$. El motivo del ingreso en la UCIP fue: en tres pacientes por EHI (42.8\%), en dos por TCE severo $(28.5 \%)$, una meningitis tuberculosa $(14.2 \%)$ y un tumor en el SNC (14.2\%). Todos los pacientes en el momento del diagnóstico presentaban un grado III de severidad (CFS).

La HSP se manifestó en un rango de 5 a 60 días de internamiento, promedio de 21.74 ( $D E \pm 18.37$ ), y el tratamiento específico se inició en un rango de 1 a 7 días, promedio de 2.00 ( $\mathrm{DE} \pm 2.07$ ). Los pacientes permanecieron en la UCIP un promedio de 22.57 días (DE \pm 10.32 ) y el tiempo de hospitalización promedio fue de 61.14 días $(D E \pm 51.24)$. Tras haber iniciado el tratamiento farmacológico, estaban asintomáticos en un rango de 3 a 10 días, promedio de $5.14(\mathrm{DE} \pm 2.17$ ). En la tabla 2 se presentan los tratamientos utilizados y la evolución de los pacientes, y en la tabla 3 se resumen las dosis de los fármacos utilizados para el control de la HSP. 


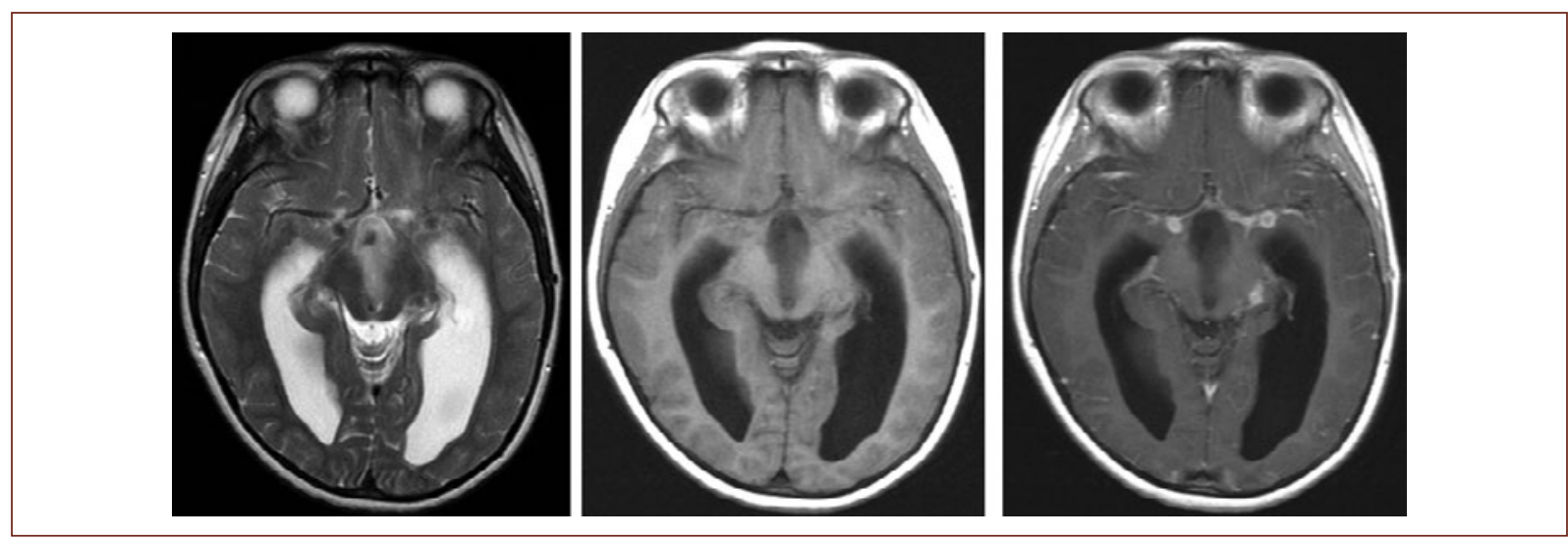

Figura 1. Imágenes axiales ponderadas en T2 y T1 simple y con gadolino a nivel del mesencéfalo, en las que se observan granulomas subaracnoideos y aracnoiditis basal que realzan con el gadolino. También hay presencia de hidrocefalia comunicante secundaria a la aracnoiditis ya descrita.

Tabla 3. Medicamentos y dosis

\begin{tabular}{|l|c|c|}
\hline Fármaco & Dosis & Vía de administración \\
\hline Baclofeno & $0.75-2 \mathrm{mg} / \mathrm{kg}$ al día & Vía oral \\
\hline PPN & $1 \mathrm{mg} / \mathrm{kg}$ al día & Vía oral \\
\hline GBP & $10 \mathrm{mg} / \mathrm{kg}$ al día & Vía oral \\
\hline BPN & $1 \mu \mathrm{g} / \mathrm{kg}$ por dosis & Intravenoso \\
\hline
\end{tabular}

En los casos 1 y 2 no se recuperaron las RM. En el expediente clínico se reportaba en el primer caso en la TAC edema cerebral severo, hematoma epidural y hemorragia intraventricular, y en el segundo caso hidrocefalia en la TAC y en la RM, que se realizó a los dos meses, con presencia de lesiones quísticas en la región occipital derecha. En la revisión de las imágenes de los otros cinco casos se encontró lo siguiente: en el caso 3, presencia de hidrocefalia comunicante por aracnoiditis infecciosa con granulomas perimesencefálicos y en los valles silvianos que realzan con el gadolinio (Fig. 1); en el caso 4, secuelas de evento hipóxico-isquémico crónico en la porción más caudal del putámen y núcleo lenticular bilateral (Fig. 2); en el paciente 5 se observaron alteraciones de señal por edema citotóxico secundario a evento hipóxico-isquémico agudo a nivel de los ganglios basales, que compromete al núcleo lenticular y al núcleo caudado bilateralmente (Fig. 3); en el caso 6 se encontró lesión axonal difusa hemorrágica y no hemorrágica en la materia blanca de ambos hemisferios cerebrales y cerebelo (Fig. 4), y en el paciente 7 se detectaron alteraciones de señal en el diencéfalo por lesión neoplásica e infartos agudos-subagudos en ambos tálamos y territorios de las arterias cerebrales posteriores (Fig. 5).

\section{Discusión}

El diagnóstico de HSP representa un reto para todo clínico, independientemente de la edad del paciente, ya que se puede confundir con otras enfermedades como síndrome neuroléptico maligno, sepsis, hipertiroidismo, feocromocitoma o hipertermia maligna, entre otras. Es un problema clínico poco frecuente que se asocia a un mal pronóstico y a un incremento en los días de internamiento ${ }^{16,17}$.

Llama la atención la baja prevalencia observada en nuestra serie, comparada con el $12 \%$ reportado en TCE $(p=1.1 \mathrm{E}-25)$ y el $29 \%$ en EHI $(p=1.6 \mathrm{E}-66)^{3,10-13 \text {; }}$ sin embargo, ofrece una muy alta significancia estadística aun con la prevalencia más baja del $6 \%$ reportada para adultos con TCE $(p=5.4 \mathrm{E}-10)^{11-13}$. Un aspecto a tener en cuenta para la baja prevalencia es el adecuado manejo en la UCIP de los factores de riesgo como hipertensión intracraneal, hipoxia y hemorragia, entre otros, que disminuyó el riesgo de desarrollar HSP; aunque otro factor pueden ser los criterios diagnósticos. Recientemente se ha propuesto una escala modificada en la que se excluyeron la sudoración y la distonía de las manifestaciones cardinales, ya que sólo se presentan en el $50 \%$ de los $\operatorname{casos}^{18}$, lo que nos hace considerar que pudieran existir formas de presentación parciales y que el padecimiento es subdiagnosticado. En nuestra serie todos los pacientes presentaron todas las manifestaciones clínicas y con un grado severo utilizando la CFS. 




Figura 2. Cortes axiales y coronales potenciados en densidad de protones y T2, en los que se observa hiperintensidad de señal a nivel de los núcleos lenticulares en la región más caudal del putamen, que representan secuelas de cambios hipóxico-isquémicos crónicos.

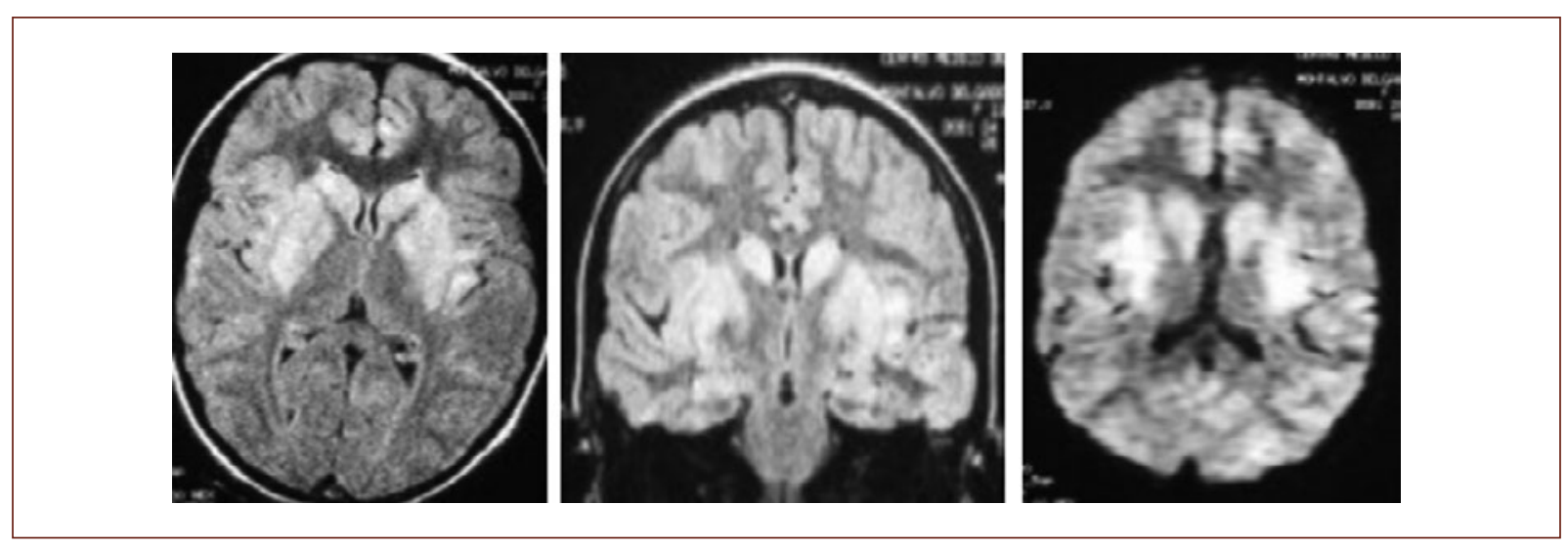

Figura 3. Cortes axiales y coronales en densidad de protones y corte axial con técnica de difusión, que muestran cambios en la intensidad de señal del núcleo lenticular y caudado bilaterales, y en ambas ínsulas, que representan edema citotóxico por cambios hipóxico-isquémicos agudos.

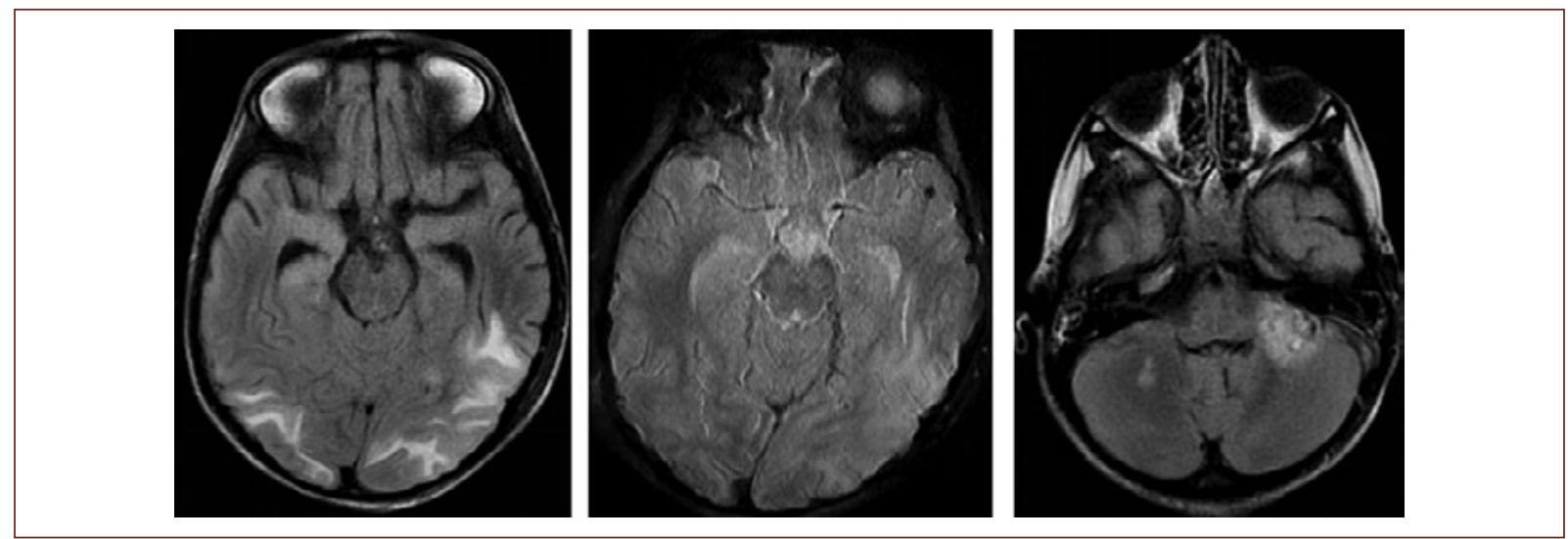

Figura 4. Imágenes axiales potenciadas en T1, densidad de protones y susceptibilidad magnética, que muestran lesión axonal difusa bilateral a nivel de la sustancia blanca yuxtacortical y lóbulos temporooccipitales bilaterales; otras son hemorrágicas subcorticales. Hemorragia parenquimatosa cerebelosa subaguda en el ángulo pontocerebeloso izquierdo y zonas de contusión no hemorrágicas en el hemisferio cerebeloso derecho. 


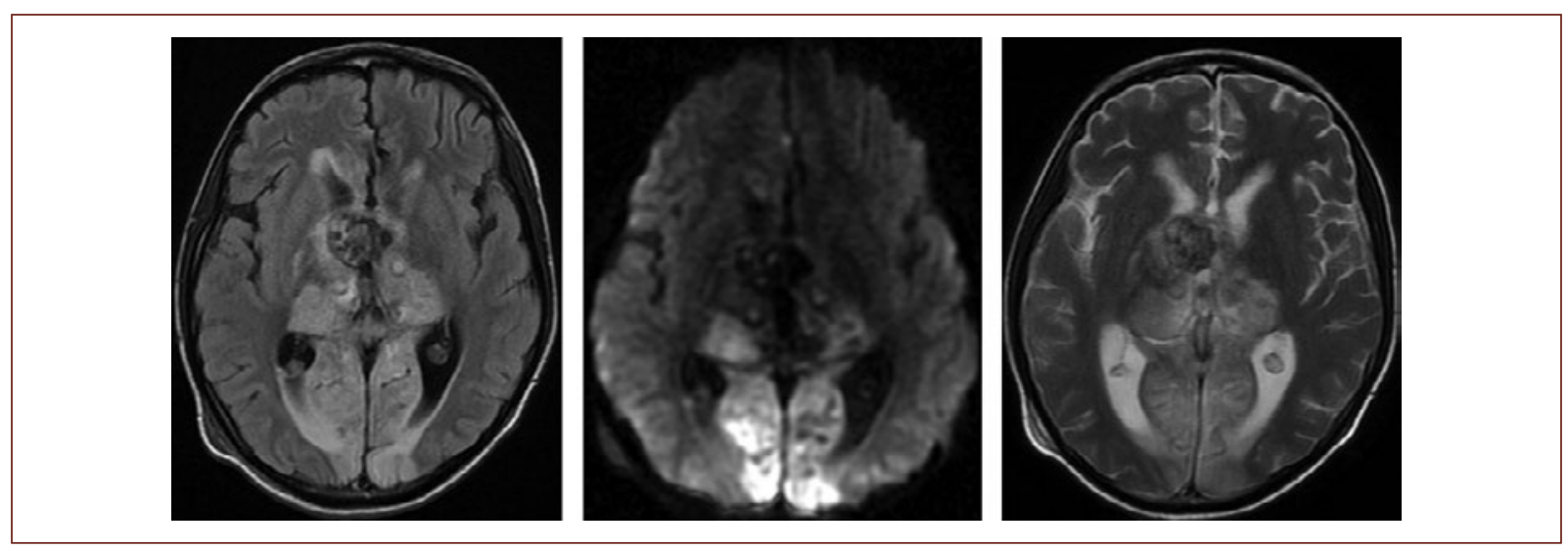

Figura 5. Imágenes axiales ponderadas en densidad de protones, difusión y T2, con lesión neoplásica en diencéfalo e infartos agudos-subagudos en ambos tálamos y lóbulos occipitales en territorios de las arterias cerebrales posteriores.

La PSH-AM ${ }^{9}$ se diseñó basándose en pacientes adultos, por lo que consideramos que se deben realizar estudios en edad pediátrica para definir los criterios diagnósticos en este grupo y validar la escala en este grupo de edad. En el trabajo de Samuel, et al. se aplicó la herramienta de forma retrospectiva en adultos, y se reportó una sensibilidad del $94 \%$ y una especificidad del $35 \%{ }^{19}$.

Al descartarse el origen epileptogénico de la HSP, han ido surgiendo diferentes teorías sobre qué estructuras están involucradas en la generación de esta respuesta disautonómica. Algunos autores han propuesto que las lesiones en el mesencéfalo provocan alteraciones en el relevo entre la médula espinal y el hipotálamo, y una de las teorías más aceptadas es el modelo propuesto por Baguley, et al., conocido como el radio excitador/inhibidor, en el que se considera que los estímulos aferentes sobre las células simpáticas localizadas en el asta posterior de la médula espinal, que han perdido su actividad normal, provocan que el centro inhibidor localizado en el diencéfalo y el tallo cerebral desencadenen una respuesta exagerada ante pequeños estímulos, con la consecuente tormenta disautonómica ${ }^{20}$.

Estudios en modelos animales han demostrado que los outputs autonómicos son regulados por la actividad de la amígdala, hipocampo, ínsula, cíngulo, corteza dorsolateral prefrontal y temporal medial, lo que hace considerar que diversas estructuras están conectadas dentro de una red autonómica central que integra la percepción visceral y las respuestas eferentes autonómicas con emociones y conductas. Estos hallazgos se han corroborado en humanos al analizar las lesiones en estudios de neuroimagen funcional, en los que se ha reportado que el principal daño se presenta en los tractos que conectan la corteza insular derecha al brazo posterior de la cápsula interna ipsilateral. Utilizando RM se han encontrado lesiones en la sustancia blanca periventricular, cuerpo calloso, diencéfalo y tronco encefálico, lo que apoya aún más la teoría de que el daño cerebral difuso es más probable que cause HSP ${ }^{15,21}$. Según la teoría de la disociación, es ocasionada por la disfunción de los centros autónomos en el diencéfalo (tálamo o hipotálamo) con sus conexiones corticales, subcorticales y tronco cerebral que median la función autonómica ${ }^{20}$. Mrkobrada, et al. reportaron en pacientes pediátricos con $\mathrm{EHI}$ afección talámica en la RM, y lo consideraron como un hallazgo temprano asociado a un peor pronóstico ${ }^{22}$. En nuestra serie no encontramos una homogeneidad de alteraciones en la neuroimagen, pues en dos casos observamos lesiones en los núcleos de la base y en un caso en el tálamo; y aunque es una serie pequeña, podemos concluir que son múltiples las áreas anatómicas involucradas en la fisiopatología de este padecimiento.

En la actualidad no se cuenta con una guía que establezca cuáles son los fármacos y las dosis eficaces. Las alternativas farmacológicas más utilizadas son morfina y benzodiacepinas intravenosas. Se ha reportado eficacia con $\alpha$-agonistas, $\beta$-bloqueantes, bromocriptina, GBP, baclofen enteral 0 intratecal ${ }^{23-28}$. En nuestra serie, en cuatro pacientes se presentó la HSP bajo sedación con midazolam. Tres pacientes respondieron con monoterapia oral (42.8\%), uno con dos fármacos enterales $(14 \%)$ y otros tres pacientes con tres fármacos $(42.8 \%)$, dos enterales y uno intravenoso. Sólo en tres pacientes se utilizó la BPN intravenosa y en un caso se tuvo que suspender el PPN por bradicardia sinusal, por lo que se reemplazó por GBP. 
A diferencia de lo que se comenta en otros trabajos, tres pacientes respondieron favorablemente con baclofeno y PPN oral, y en los tres últimos casos se utilizó la BPN, ya que anteriormente no contábamos con el fármaco en nuestra institución. A pesar de que no se encontró significancia estadística entre los pacientes que utilizaron BPN y fármacos orales, se observó que los días de internamiento en el grupo de BPN fue de 26 versus $87.5(p=0.25)$ y los días en la UCIP de 19.3 versus 25 ( $p=0.39$ ); sin embargo, los días sin HSP fue menor en el grupo sin BPN, con 4.75 versus 5.6 $(p=0.35)$. En el caso de la NI, observamos que con la aplicación de la dexametasona (DEX) intratecal la paciente presentó mejoría del cuadro clínico.

Aunque hay muy pocos trabajos, se ha informado que el uso de la dexmedetomidina, $\alpha_{2}$-agonista selectivo de receptores, disminuye el riesgo en los pacientes postoperados de TCE para desarrollar HSP, en comparación con otros sedantes; aunque los mismos autores concluyen que no ayuda a disminuir las secuelas neurológicas en este grupo de pacientes ${ }^{29,30}$.

Dentro de los cuidados generales se deben evitar los estímulos que desencadenen las manifestaciones autonómicas, por lo que se recomienda evitar el ruido y los estímulos luminosos intensos, permitir pocas visitas al paciente y disminuir al máximo el estímulo táctil, como evitando los baños con agua fría, una movilización innecesaria, no utilizar ropa ajustada y prevenir la constipación $^{31,32}$. La terapia física es muy importante en este padecimiento para ayudar a la recuperación de los pacientes. Ésta debe ser multidisciplinaria, aunque el problema surge a la hora de determinar con qué tipo de terapia empezar, ya que en etapas tempranas, como ya se ha mencionado, los estímulos pueden precipitar las crisis $^{33}$. Seis pacientes de nuestro estudio han continuado con la terapia física y en vigilancia neurológica en nuestra institución. Según la escala de Rankin modificada a un año del padecimiento, tenemos a tres pacientes con nivel 1 , a dos pacientes con nivel 5 y a dos más con nivel 6 . El paciente 7 falleció a las tres semanas de la HSP por complicaciones propias de la neoplasia. Tres de los pacientes reciben antiepilépticos, con lo que se ha logrado un control de la epilepsia. El paciente 1 falleció después del año, secundario a sepsis posterior a la colocación de placa craneal. En nuestra serie, a diferencia de lo reportado en otros trabajos ${ }^{3,34}$, en un rango de 3 a 10 días después del inicio de fármacos para la HSP los pacientes se encontraban asintomáticos y no se reportaron manifestaciones simpáticas al estimularlos durante la terapia física. A todos los pacientes se les suspendió a los seis meses los fármacos para HSP, sin recaída en ningún caso.

\section{Conclusiones}

La HSP es un padecimiento grave y su reconocimiento temprano es muy importante, pues los principales objetivos son iniciar un tratamiento de inmediato, y evitar las complicaciones y la realización de estudios innecesarios.

Según nuestra opinión, se deben realizar revisiones sistemáticas del trastorno en edad pediátrica con los objetivos de establecer si las manifestaciones clínicas son iguales que en los adultos y para elaborar guías de tratamiento para pacientes pediátricos.

\section{Conflicto de intereses}

Los autores declaran no tener ningún conflicto de intereses.

\section{Fuentes de financiamiento}

No se recibió ningún financiamiento para la realización del trabajo.

\section{Bibliografía}

1. Blackman JA, Patrick PD, Buck ML, Rust RS Jr. Paroxysmal autonomic instability with dystonia after brain injury. Arch Neurol. 2004;61(3):321-8.

2. Perkes I, Baguley IJ, Nott MT, Menon DK. A review of paroxysmal sympathetic hyperactivity after acquired brain injury. Ann Neurol. 2010;68(2): 126-35.

3. Kirk KA, Shoykhet M, Jeong JH, Tyler-Kabara EC, Henderson MJ, Bell MJ, et al. Dysautonomia after pediatric brain injury. Dev Med Child Neurol. 2012;54(8):759-64.

4. Goh KY, Conway EJ, DaRosso RC, Muszynski CA, Epstein FJ. Sympathetic storms in a child with a midbrain glioma: a variant of diencephalic seizures. Pediatr Neurol. 1999;21(4):742-4.

5. Ramdhani NA, Sikma MA, Witkamp TD, Slooter AJ, de Lange DW. Paroxysmal autonomic instability with dystonia in a patient with tuberculous meningitis: a case report. J Med Case Rep. 2010;4:304.

6. Farias-Moeller R, Carpenter JL, Dean N, Wells EM. Paroxysmal Sympathetic Hyperactivity in Critically III Children with Encephalitis and Meningoencephalitis. Neurocrit Care. 2015;23(3):380-5.

7. Hinson HE, Takahashi C, Altowaijri G, Baguley IJ, Bourdette D. Anti-NMDA receptor encephalitis with paroxysmal sympathetic hyperactivity: an under-recognized association? Clin Auton Res. 2013;23(2):109-11.

8. Strich SJ. Diffuse degeneration of the cerebral white matter in severe dementia following head injury. I Neurol Neurosurg Psychiatry. 1956;19(3):163-85.

9. Baguley IJ, Perkes IE, Fernández-Ortega JF, Rabinstein AA, Dolce G, Hendricks HT. Paroxysmal sympathetic hyperactivity after acquired brain injury: consensus on conceptual definition, nomenclature, and diagnostic criteria. J Neurotrauma. 2014;31(17):1515-20.

10. Krach LE, Kriel RL, Morris WF, Warhol BL, Luxenberg MG. Central autonomic dysfunction following acquired brain injury in children. Neurorehabil Neural Repair. 1997;11:41-5.

11. Baguley IJ, Slewa-Younan S, Heriseanu RE, Nott MT, Mudaliar Y, Nayyar $\mathrm{V}$. The incidence of dysautonomia and its relationship with autonomic arousal following traumatic brain injury. Brain Inj. 2007;21(11):1175-81.

12. Rabinstein AA. Paroxysmal sympathetic hyperactivity in the neurological intensive care unit. Neurol Res. 2007;29(7):680-2.

13. Hinson HE, Sheth KN. Manifestations of the hyperadrenergic state after acquired brain injury. Curr Opin Crit Care. 2012;18(2):139-45. 


\section{A. Bravo-Oro, et al.: Hiperactividad simpática paroxística en pacientes pediátricos}

14. Meyer KS. Understanding paroxysmal sympathetic hyperactivity after traumatic brain injury. Surg Neurol Int. 2014;5(Suppl 13):S490-2.

15. Hinson HE, Puybasset L, Weiss N, Perlbarg V, Benali H, Galanaud D, et al. Neuroanatomical basis of paroxysmal sympathetic hyperactivity: a diffusion tensor imaging analysis. Brain Inj. 2015;29(4):455-61.

16. Fernández-Ortega JF, Prieto-Palomino MA, García-Caballero $M$, Galeas-López JL, Quesada-García G, Baguley IJ. Paroxysmal sympathetic hyperactivity after traumatic brain injury: clinical and prognostic implications. J Neurotrauma. 2012;29(7):1364-70.

17. Bueno González AM, Corcobado Márquez MC, Portilla Botelho M, Ambrós Checa A. Diagnostic challenge of paroxysmal sympathetic hyperactivity (PSH) associated with diffuse axonal injury (DAI) in head trauma. Springerplus. 2014;3:752.

18. Hinson HE, Schreiber MA, Laurie AL, Baguley IJ, Bourdette D, Ling GS Early fever as a predictor of paroxysmal sympathetic hyperactivity in traumatic brain injury. J Head Trauma Rehabil. 2017;32(5):E50-4.

19. Samuel S, Lee M, Brown RJ, Choi HA, Baguley IJ. Incidence of paroxysmal sympathetic hyperactivity following traumatic brain injury using assessment tools. Brain Inj. 2018;32(9):1115-21.

20. Baguley IJ. The excitatory: inhibitory ratio model (EIR Model): an integrative explanation of acute automonic overactivity syndromes. Med Hypotheses. 2008;70(1):26-35.

21. Lv LQ, Hou LJ, Yu MK, Qi XQ, Chen HR, Chen JX, et al. Prognostic influence and magnetic resonance imaging findings in paroxysmal sympathetic hyperactivity after severe traumatic brain injury. J Neurotrauma. 2010;27(11):1945-50.

22. Mrkobrada S, Wei XC, Gnanakumar V. Magnetic resonance imaging findings of bilateral thalamic involvement in severe paroxysmal sympathetic hyperactivity: a pediatric case series. Childs Nerv Syst. 2016;32(7): 1299-303.

23. Hughes JD, Rabinstein AA. Early diagnosis of paroxysmal sympathetic hyperactivity in the ICU. Neurocrit Care. 2014;20(3):454-9.

24. Feng $Y$, Zheng $X$, Fang $Z$. Treatment progress of paroxysmal sympathetic hyperactivity after acquired brain injury. Pediatr Neurosurg. 2015;50(6): $301-9$
25. Raithel DS, Ohler KH, Porto I, Bicknese AR, Kraus DM. Morphine: an effective abortive therapy for pediatric paroxysmal sympathetic hyperactivity after hypoxic brain injury. J Pediatr Pharmacol Ther. 2015;20(4): 335-40.

26. Samuel S, Allison TA, Lee K, Choi HA. Pharmacologic management of paroxysmal sympathetic hyperactivity after brain injury. J Neurosci Nurs. 2016;48(2):82-9.

27. Burton JM, Morozova OM. Calming the storm: dysautonomia for the pediatrician. Curr Probl Pediatr Adolesc Health Care. 2017:47(7):145-50.

28. Pozzi M, Conti V, Locatelli F, Galbiati S, Radice S, Clementi E, et al. Paroxysmal sympathetic hyperactivity in pediatric rehabilitation: pathological features and scheduled pharmacological therapies. J Head Trauma Rehabil. 2017;32(2):117-24.

29. Tang $Q$, Wu X, Weng W, Li H, Feng J, Mao $Q$, et al. The preventive effect of dexmedetomidine on paroxysmal sympathetic hyperactivity in severe traumatic brain injury patients who have undergone surgery: a retrospective study. PeerJ. 2017;5:e2986.

30. Peng Y, Zhu H, Chen H, Zhu Z, Zhou H, Zhang S, et al. Dexmedetomidine attenuates acute paroxysmal sympathetic hyperactivity. Oncotarget. 2017;8(40):69012-9.

31. Letzkus L, Keim-Malpass J, Anderson J, Kennedy C. Paroxysmal sympathetic hyperactivity in children: an exploratory evaluation of nursing interventions. J Pediatr Nurs. 2017;34:e17-21.

32. Letzkus L, Addison N, Turner L, Conaway M, Quatrara B. Paroxysmal sympathetic hyperactivity and environmental factors: a pilot study. J Neurosci Nurs. 2018;50(2):88-92.

33. Thomas A, Greenwald BD. Paroxysmal sympathetic hyperactivity and clinical considerations for patients with acquired brain injuries: a narrative review. Am J Phys Med Rehabil. 2018. [Epub ahead of print]

34. Baguley IJ, Heriseanu RE, Nott MT, Chapman J, Sandanam J. Dysautonomia after severe traumatic brain injury: evidence of persisting overresponsiveness to afferent stimuli. Am J Phys Med Rehabil. 2009;88(8): $615-22$ 